\title{
THE BIDDER'S CURSE: COMMENT
}

Henry S. Schneider

March 2014

\section{ONLINE APPENDIX}

I provide several additional tables below. Tables A1, A2, and A5, and Figure A1 show the results from the main text using data that exclude shipping fees. Tables A3 and A4 show the outliers in the M\&L cross section with and without shipping fees. The mismatches and reasons for mismatches, referred to in Footnote 17 of the main text, are at the end of this document.

Table A1: Wording differences and overbidding in cross section, excluding shipping fees

\begin{tabular}{lcccccc}
\hline & & & & & & $\Delta$ price \\
& & Fraction & $\Delta$ price & $\Delta$ price & $\begin{array}{c}\text { mean } \\
\text { ex-outliers } \\
\text { (percent) }\end{array}$ \\
\hline Auction has "new," BIN does not & 269 & 0.62 & 0.51 & 9.50 & 5.11 & 10.07 \\
Auction, BIN have or do not have "new" & 1,045 & 0.45 & 0.37 & 34.66 & -2.56 & 4.10 \\
Auction does not have "new," BIN does & 377 & 0.43 & 0.34 & 0.01 & -4.60 & 1.49 \\
\hline All auctions & 1,691 & 0.47 & 0.39 & 22.95 & -1.49 & 4.48 \\
\hline
\end{tabular}

Notes: "Fraction overbid" and "Fraction overbid 5 percent" are the fraction of auctions that end above the low BIN price, and the fraction of auctions that end over 5 percent above the low BIN price, respectively. " $\Delta$ price mean (percent)" is the mean percentage difference between the auction ending price and the low BIN price across products. " $\Delta$ price median (percent)" is the median percentage difference, which reduces the influence of outliers. " $\Delta$ price mean ex-outliers (percent)" is another approach for limiting the influence of outliers, by dropping the bottom and top 1 percent of products based on the percentage difference between the auction and low-price BIN. The bottom and top 1 percent of outliers are in Table A3 below. Prices exclude shipping fees. 
Table A2: Movies, prices, and overbidding rates in DVD data set, excluding shipping fees

\begin{tabular}{|c|c|c|c|c|c|c|c|}
\hline \multirow[b]{2}{*}{ Movie Title } & \multirow[b]{2}{*}{ Version } & \multirow[b]{2}{*}{$\mathrm{N}$} & \multirow{2}{*}{$\begin{array}{c}\text { Mean } \\
\text { auction } \\
\text { price }(\$)\end{array}$} & \multirow{2}{*}{$\begin{array}{c}\text { Mean } \\
\text { low BIN } \\
\text { price }(\$)\end{array}$} & \multirow{2}{*}{$\begin{array}{l}\text { Fraction } \\
\text { overbid }\end{array}$} & \multicolumn{2}{|c|}{$\begin{array}{c}\text { Auction - BIN } \\
\text { price }(\$)\end{array}$} \\
\hline & & & & & & Mean & SE \\
\hline \multirow[t]{2}{*}{ Batman Begins } & Regular & 35 & 4.49 & 3.57 & 0.69 & 0.92 & 0.39 \\
\hline & Special & 1 & 4.78 & 13.69 & 0.00 & -8.91 & \\
\hline Camp Rock & Regular & 97 & 10.26 & 12.23 & 0.31 & -1.98 & 0.31 \\
\hline \multirow[t]{2}{*}{ Casino Royale } & Regular & 18 & 6.87 & 7.86 & 0.22 & -0.99 & 0.69 \\
\hline & Special & 3 & 16.18 & 25.48 & 0.00 & -9.30 & 3.26 \\
\hline College Road Trip & Regular & 30 & 5.13 & 6.52 & 0.20 & -1.40 & 0.44 \\
\hline $\begin{array}{l}\text { Harold and Kumar Escape } \\
\text { From Guantanamo Bay }\end{array}$ & Regular unrated & 10 & 6.30 & 15.56 & 0.00 & -9.26 & 1.45 \\
\hline \multirow[t]{2}{*}{ Knocked Up } & Regular unrated & 34 & 3.78 & 6.28 & 0.18 & -2.50 & 0.42 \\
\hline & Special unrated & 1 & 10.50 & 24.47 & 0.00 & -13.97 & \\
\hline \multirow[t]{2}{*}{ Live Free or Die Hard } & Regular unrated & 9 & 7.42 & 5.63 & 0.44 & 1.78 & 2.18 \\
\hline & Special unrated & 1 & 9.00 & 11.98 & 0.00 & -2.98 & . \\
\hline $\begin{array}{l}\text { Miss Pettigrew Lives } \\
\text { For a Day }\end{array}$ & Regular & 16 & 8.33 & 13.22 & 0.06 & -4.89 & 0.61 \\
\hline \multirow[t]{2}{*}{ Pirates of the Caribbean 3} & Regular & 63 & 5.17 & 6.92 & 0.21 & -1.75 & 0.30 \\
\hline & Special & 1 & 12.55 & 14.95 & 0.00 & -2.40 & . \\
\hline Riddick Trilogy & Regular & 4 & 7.69 & 7.20 & 0.50 & 0.49 & 4.49 \\
\hline Shark Tale & Regular & 6 & 6.63 & 4.95 & 0.83 & 1.68 & 1.36 \\
\hline \multirow[t]{2}{*}{ Street Kings } & Regular & 39 & 6.03 & 7.61 & 0.28 & -1.58 & 0.37 \\
\hline & Special & 7 & 7.58 & 10.34 & 0.14 & -2.76 & 1.27 \\
\hline \multirow[t]{2}{*}{ The Bank Job } & Regular & 27 & 4.58 & 4.00 & 0.67 & 0.59 & 0.85 \\
\hline & Special & 2 & 9.99 & 18.39 & 0.00 & -8.40 & 6.39 \\
\hline The Notebook & Regular & 30 & 8.91 & 10.79 & 0.17 & -1.87 & 0.35 \\
\hline The Scorpion King 2 & Regular & 50 & 4.41 & 6.95 & 0.12 & -2.54 & 0.28 \\
\hline \multirow[t]{3}{*}{ Transformers } & Regular & 37 & 7.15 & 11.02 & 0.11 & -3.87 & 0.65 \\
\hline & Special & 31 & 9.34 & 11.34 & 0.29 & -2.00 & 0.90 \\
\hline & & 552 & 6.84 & 8.80 & 0.27 & -1.96 & 0.16 \\
\hline
\end{tabular}

Notes: Shown are statistics for each movie title and version that resulted in sale in the observational data set. "Fraction overbid" is the fraction of auctions with an ending price that exceeds the corresponding lowest-price BIN. "Auction - BIN price (\$)" is the difference between the mean auction price and the mean low BIN price. Prices do not include shipping fees. 


\begin{tabular}{|c|c|c|c|}
\hline Auction title & BIN title & $\begin{array}{l}\text { Auction } \\
\text { price }\end{array}$ & $\begin{array}{l}\mathrm{BIN} \\
\text { price }\end{array}$ \\
\hline NEW CANON POWERSHOT A630 DIGITAL CAMERA 8.0MP 4x ZOOM & Canon PowerShot A630 8.0 Megapixel Brand new In Box & 199.29 & 19.99 \\
\hline NEW Braun 360 Complete 8995 Shaver -NO RESERVE! !! & BRAUN 360 COMPLETE 8995 SHAVER BRAND NEW SEALED & 157 & 61 \\
\hline The Secret by Rhonda Byrne (2006) & The Secret by Rhonda Byrne (2006) & 32.5 & 13.5 \\
\hline The Secret by Rhonda Byrne (2006) As seen on Oprah & The Secret by Rhonda Byrne (2006) & 25.99 & 10.98 \\
\hline The Secret by Rhonda Byrne (2006) NEW Hardcover & The Secret by Rhonda Byrne (2006) & 34 & 16.5 \\
\hline The Secret by Rhonda Byrne BRAND NEW BOOK!! HARDCOVER & The Secret by Rhonda Byrne (2006) & 24.49 & 11.98 \\
\hline$\sim$ THE SECRET $\sim$ by Rhonda Byrne BRAND NEW - Hardcover & The Secret by Rhonda Byrne (2006) & 24.48 & 11.98 \\
\hline Canon PowerShot A630 8.0 Megapixel Brand new In Box & Canon PowerShot A630 8.0 Megapixel DIGITAL Camera & 179.5 & 89 \\
\hline Quicken Home and Business 2007 FACTORY SEALED & Quicken personal finances 2007 Home \& business NEW & 58.06 & 30 \\
\hline The Secret by Rhonda Byrne (2006) & The Secret by Rhonda Byrne (2006) & 20.99 & 10.98 \\
\hline The Secret by Rhonda Byrne (2006) & The Secret by Rhonda Byrne (2006) & 31.5 & 16.5 \\
\hline NEW! The Secret by Rhonda Byrne Hardcover (2006) - NEW & The Secret by Rhonda Byrne (2006) & 30.55 & 16.5 \\
\hline NEW Apple iPod I POD Nano Blue 4GB MP3 & New Blue Ipod Nano 4GB- Factory Sealed -Free Shipping & 321 & 175 \\
\hline THE SECRET by Rhonda Byrne (As Seen On Oprah) NEW HC & The Secret by Rhonda Byrne (2006) & 30 & 16.5 \\
\hline Proactiv Renewing Cleanser 4 oz NEW SEALED Proactive 08 & Proactiv Renewing Cleanser 4 oz NEW SEALED Proactive 08 & 6.83 & 12.98 \\
\hline OralB Professional Care 7850 DLX & Oral-B PROFESSIONALCARE 7850-DLX NEW \& SEALED! & 22.25 & 42.98 \\
\hline Culture Warrior by Bill O'Reilly (2006)NEW H/C/D/J & Culture Warrior by Bill O'Reilly HC/DJ & 9.49 & 18.5 \\
\hline BUMBLE AND BUMBLE Bb TONIC LOTION - 8 OZ. NEW & 8 oz. Bumble and Bumble Tonic Lotion. NEW. & 12.5 & 24.44 \\
\hline H\&R Block TaxCut Premium Federal/State 2006 Win/Mac & NEW H\&R Block TaxCut Premium Federal + State 2006 & 8.5 & 17.09 \\
\hline D-LINK DI-524 WIRELESS G54 ROUTER (NIB) NEVER OPEN & D-LINK AirPlus ${ }^{\mathrm{a}}$ G DI-524 HIGH SPEED WIRELESS ROUTER & 35.5 & 75.74001 \\
\hline Omron HJ-112 Pedometer & New OMRON HJ-112 HJ112 WALKING PEDOMETER & 10.5 & 22.44 \\
\hline ISLAND KISS BY ESCADA 3.4 FL OZ EDT & NEW ESCADA Island Kiss Women Perfume 3.4 Fl Oz & 16.6 & 35.94 \\
\hline D-Link DI-524 Wireless G Router AirPlus G/NIB Sealed & D-LINK AirPlus ${ }^{\mathrm{a}}$ G DI-524 HIGH SPEED WIRELESS ROUTER & 34.5 & 75.74001 \\
\hline Bones Reds Skateboard Bearings set of 8 FREE Ship US & Powell Bones Reds Skateboard Bearings (Set of 8) & 9 & 20.04 \\
\hline Quicken Home \& Business 2007 - Brand New \& Factory Seal & Quicken Home And Business 2007 Full Retail Box & 18.52 & 44.95 \\
\hline BONES REDS SKATEBOARD BEARINGS set of 8 FREE SHIP US & Powell Bones Reds Skateboard Bearings (Set of 8) & 7.53 & 20.04 \\
\hline NEW Apple iPod I POD Nano Blue 4GB MP3 & New Blue Ipod Nano 4GB- Factory Sealed -Free Shipping & 53 & 175 \\
\hline Apple Ipod video (80GB), Black Digital Media Player & Apple Ipod Video (80GB), Black Digital Media Player & 80 & 325 \\
\hline
\end{tabular}

Notes: The bottom and top one percent of auction-BIN pairs based on the auction-BIN percentage price differences are shown. This table does not include mismatches. Shipping fees are included. 


\begin{tabular}{|c|c|c|c|}
\hline Auction title & BIN title & $\begin{array}{l}\text { Auction } \\
\text { price }\end{array}$ & $\begin{array}{l}\text { BIN } \\
\text { price }\end{array}$ \\
\hline －!New ORANGE Apple iPod Shuffle (1GB) MP3 NR!- & APPLE IPOD Orange Shuffle 1GB MP3 Player : BRAND NEW & 66.00 & 0.49 \\
\hline Orange Apple IPod Shuffle New in Box NIB & APPLE IPOD Orange Shuffle 1GB MP3 Player : BRAND NEW & 65.00 & 0.49 \\
\hline NEW CANON POWERSHOT A630 DIGITAL CAMERA 8.0MP 4x ZOOM & Canon PowerShot A630 8.0 Megapixel Brand new In Box & 179.30 & 9.99 \\
\hline Canon PowerShot A630 8.0 Megapixel BRAND NEW IN BOX! & Canon PowerShot A630 8.0 Megapixel Digital Camera NEW & 172.51 & 10.50 \\
\hline Canon PowerShot A630 8.0 Megapixel Brand new In Box & Canon PowerShot A630 8.0 Megapixel Digital Camera NEW & 169.50 & 31.30 \\
\hline Omron Digital Premium Pedometer HJ-112 (New in Box) & NEW IN BOX Omron HJ-112 Digital Premium Pedometer HJ112 & 20.00 & 4.98 \\
\hline * NEW * Omron Premium Pedometer Model HJ-112 & NEW IN BOX Omron HJ-112 Digital Premium Pedometer HJ112 & 18.50 & 4.98 \\
\hline Brand New Omron Premium Digital Pedometer HJ-112 HJ112 & NEW IN BOX Omron HJ-112 Digital Premium Pedometer HJ112 & 17.50 & 4.98 \\
\hline ** Omron HJ-112 Digital Premium Pedometer Brand New ** & NEW IN BOX Omron HJ-112 Digital Premium Pedometer HJ112 & 16.95 & 4.98 \\
\hline ** Omron HJ-112 Digital Premium Pedometer Brand New ** & NEW IN BOX Omron HJ-112 Digital Premium Pedometer HJ112 & 16.95 & 4.98 \\
\hline Brand New Omron Premium Digital Pedometer HJ-112 HJ112 & NEW IN BOX Omron HJ-112 Digital Premium Pedometer HJ112 & 15.00 & 4.98 \\
\hline Omron HJ-112 Digital Workout Pedometer - BRAND NEW & NEW IN BOX Omron HJ-112 Digital Premium Pedometer HJ112 & 14.99 & 4.98 \\
\hline NEW Omron Premium Pedometer HJ-112 Step Counter & NEW IN BOX Omron HJ-112 Digital Premium Pedometer HJ112 & 14.99 & 4.98 \\
\hline The Secret by Rhonda Byrne (2006) NEW Hardcover & THE SECRET by Rhonda Byrne (2006) BRAND NEW & 29.00 & 9.95 \\
\hline NEW OMRON HJ-112 Pedometer Step Calorie Walking Counter & NEW IN BOX Omron HJ-112 Digital Premium Pedometer HJ112 & 14.50 & 4.98 \\
\hline NEW Braun 360 Complete 8995 Shaver -NO RESERVE! !! & BRAUN 360 COMPLETE 8995 SHAVER BRAND NEW SEALED & 147 & 51 \\
\hline Culture Warrior by Bill O'Reilly (2006) NEW HC & Culture Warrior by Bill O'Reilly HC/DJ & 4.75 & 14.00 \\
\hline Omron HJ-112 Pedometer & NEW IN BOX Omron HJ-112 Digital Premium Pedometer HJ112 & 3.00 & 8.98 \\
\hline Belkin Wireless G Router - Brand new in a Box & Belkin (F5D7230-4) 54Mbps Wireless G Router & 9.99 & 29.95 \\
\hline You on a Diet by Craig Wynett, Lisa Oz, Mehmet C. Oz... & You on a Diet by Craig Wynett, Lisa Oz, Mehmet C. Oz... & 3.25 & 9.88 \\
\hline Proactiv Renewing Cleanser 4 oz NEW SEALED Proactive 08 & Proactiv Renewing Cleanser 4 oz NEW SEALED Proactive 08 & 2.84 & 8.99 \\
\hline ISLAND KISS BY ESCADA 3.4 FL OZ EDT & Escada Island Kiss 3.4oz Eau De Toilette NEW IN BOX & 7.61 & 28.99 \\
\hline In Line Spark Plug Tester Tool Test Ignition System & Inline Ignition Spark Plug / Wire / Coil Tester & 0.99 & 4.25 \\
\hline Apple Ipod video (80GB), Black Digital Media Player & Apple Ipod Video (80GB), Black Digital Media Player & 68.00 & 312.99 \\
\hline NEW Apple iPod I POD Nano Blue 4GB MP3 & APPLE IPOD NANO (4GB) 2nd GENERATION BLUE - NEW & 27.00 & 174.00 \\
\hline The Audacity of Hope by Barack Obama (2006) & The Audacity of Hope by Barack Obama (2006) & 0.99 & 6.89 \\
\hline H\&R Block Tax Cut 2006 Premium Federal + State & Brand New!! H\&R Block TaxCut Premium Federal+State 2006 & 1.04 & 9.50 \\
\hline TI 83 Plus Graphing Calculator Book, Cable, \& Batteries & Texas Instruments TI-83 Plus Graphing Calculator New & 4.99 & 61.00 \\
\hline IN-LINE IGNITION SPARK PLUG TESTER TROUBLE SHOOTER & NEW INLINE IGNITION SPARK PLUG HT TESTER TOOL & 0.01 & 3.95 \\
\hline IN-LINE IGNITION SPARK PLUG TESTER $\sim$ TROUBLE SHOOTER & NEW INLINE IGNITION SPARK PLUG HT TESTER TOOL & 0.01 & 3.95 \\
\hline IN-LINE IGNITION SPARK PLUG TESTER TROUBLE SHOOTER & Inline Ignition Spark Plug / Wire / Coil Tester & 0.01 & 4.25 \\
\hline In Line Spark Plug Tester Tool Test Ignition System NR & Inline Ignition Spark Plug / Wire / Coil Tester & 0.01 & 4.25 \\
\hline
\end{tabular}

Notes: The bottom and top one percent of auction-BIN pairs based on the auction-BIN percentage price differences are shown. This

table does not include mismatches. Shipping fees are excluded. Table A3 has more observations than Table A2 because the data set without shipping fees is slightly larger due to some observations missing shipping fee data. 
Table A5: Auction-BIN price differences in cross section, excluding shipping fees

\begin{tabular}{|c|c|c|c|c|c|c|c|}
\hline Item & $\mathrm{N}$ & $\begin{array}{r}\text { Fraction } \\
\text { overbid }\end{array}$ & $\begin{array}{c}\Delta \text { price } \\
\text { mean } \\
\text { (percent) }\end{array}$ & Item & $\mathrm{N}$ & $\begin{array}{r}\text { Fraction } \\
\text { overbid }\end{array}$ & $\begin{array}{c}\Delta \text { price } \\
\text { mean } \\
\text { (percent) }\end{array}$ \\
\hline $\begin{array}{l}\text { Western Digital My } \\
\text { Book 500GB }\end{array}$ & 26 & 0.31 & -2 & $\begin{array}{l}\text { Lancome Definicils } \\
\text { mascara }\end{array}$ & 13 & 0.31 & -5 \\
\hline $\begin{array}{l}\text { Sandisk Secure Digital } \\
\text { Ultra } 4 \text { GB }\end{array}$ & 15 & 0.40 & -11 & Murad & 13 & 0.00 & -18 \\
\hline Quicken 2007 Basic & 41 & 0.44 & -3 & $\begin{array}{l}\text { Farouk Chi iron } \\
\text { straightener ceramic }\end{array}$ & 34 & 0.21 & -5 \\
\hline Quicken 2007 Deluxe & 10 & 0.40 & -8 & Omron 112 pedometer & 27 & 0.93 & 111 \\
\hline $\begin{array}{l}\text { Quicken } 2007 \text { Home } \\
\text { Business }\end{array}$ & 39 & 0.72 & 20 & $\begin{array}{l}\text { Vitality Sonic Oral } \\
\text { electronic toothbrush }\end{array}$ & 15 & 0.20 & -21 \\
\hline $\begin{array}{l}\text { H\&R Block Federal and } \\
\text { State }\end{array}$ & 44 & 0.73 & 15 & $\begin{array}{l}\text { Sonic S-320 electronic } \\
\text { toothbrush }\end{array}$ & 15 & 0.07 & -27 \\
\hline $\begin{array}{l}\text { Braun Electric Shaver } \\
8995\end{array}$ & 15 & 0.53 & 18 & $\begin{array}{l}\text { Oral-B } 7850 \text { DLX } \\
\text { electronic toothbrush }\end{array}$ & 17 & 0.29 & -6 \\
\hline $\begin{array}{l}\text { Braun } 360 \text { Electric } \\
\text { Shaver } 8985\end{array}$ & 38 & 0.58 & 0 & $\begin{array}{l}\text { Oral-B } 9400 \text { Triumph } \\
\text { electronic toothbrush }\end{array}$ & 56 & 0.52 & 1 \\
\hline $\begin{array}{l}\text { D-LINK DI-524 wireless } \\
\text { router }\end{array}$ & 12 & 0.17 & -22 & $\begin{array}{l}\text { Sonicare } 7300 \text { electronic } \\
\text { toothbrush }\end{array}$ & 17 & 0.41 & 1 \\
\hline $\begin{array}{l}\text { Linksys WRT300N } \\
\text { wireless router }\end{array}$ & 22 & 0.09 & -15 & Zune 30GB black & 34 & 0.50 & 1 \\
\hline $\begin{array}{l}\text { Calvin Klein Eternity } \\
\text { cologne }\end{array}$ & 13 & 0.15 & -12 & Zune $30 \mathrm{~GB}$ white & 15 & 0.33 & -4 \\
\hline $\begin{array}{l}\text { Calvin Klein Eternity } \\
\text { perfume }\end{array}$ & 20 & 0.60 & 7 & Roomba Scheduler 4230 & 21 & 0.33 & -5 \\
\hline $\begin{array}{l}\text { SixAxis Wireless PS3 } \\
\text { controller }\end{array}$ & 26 & 0.31 & -5 & $\begin{array}{l}\text { Bumble and Bumble } \\
\text { Hair Tonic }\end{array}$ & 13 & 0.00 & -41 \\
\hline $\begin{array}{l}\text { Wireless Xbox } 360 \\
\text { controller }\end{array}$ & 26 & 0.92 & 21 & $\begin{array}{l}\text { TI-89 Titanium graphing } \\
\text { calculator }\end{array}$ & 30 & 0.33 & -7 \\
\hline $\begin{array}{l}\text { Motorola Razr D\&G } \\
\text { gold cell phone }\end{array}$ & 12 & 0.75 & 5 & $\begin{array}{l}\text { TI-83 Plus graphing } \\
\text { calculator }\end{array}$ & 25 & 0.32 & -12 \\
\hline $\begin{array}{l}\text { Garmin GPS C330 } \\
\text { navigation system }\end{array}$ & 14 & 0.07 & -11 & Sandisk Cruzer Micro & 29 & 0.03 & -23 \\
\hline TMX Elmo doll & 76 & 0.25 & -14 & $\begin{array}{l}\text { Scrubs complete season } \\
\text { DVDs }\end{array}$ & 20 & 0.60 & 1 \\
\hline You on a Diet (book) & 97 & 0.51 & 0 & $\begin{array}{l}\text { Proactive Renewing } \\
\text { Cleanser }\end{array}$ & 17 & 0.29 & -11 \\
\hline $\begin{array}{l}\text { The Audicity of Hope } \\
\text { (book) }\end{array}$ & 20 & 0.40 & -11 & Canon A630 camera & 10 & 0.30 & 358 \\
\hline Culture Warrior (book) & 21 & 0.33 & -22 & T-Mobile Sidekick 3 & 17 & 0.29 & -2 \\
\hline The Secret (book) & 181 & 0.91 & 58 & Lost First Season DVDs & 20 & 0.80 & 14 \\
\hline $\begin{array}{l}\text { The Best Life Diet } \\
\text { (book) }\end{array}$ & 68 & 0.76 & 17 & $\begin{array}{l}\text { Grey's Anatomy Second } \\
\text { Season DVDs }\end{array}$ & 11 & 0.18 & -13 \\
\hline $\begin{array}{l}\text { iPod Shuffle 2nd } \\
\text { generation blue }\end{array}$ & 17 & 0.47 & 1 & $\begin{array}{l}\text { Lost Second Season } \\
\text { DVDs }\end{array}$ & 21 & 0.86 & 25 \\
\hline $\begin{array}{l}\text { iPod Shuffle } 2 \text { nd } \\
\text { generation orange }\end{array}$ & 12 & 0.58 & 2210 & $\begin{array}{l}\text { Belkin F5D7230 } \\
\text { wireless router }\end{array}$ & 12 & 0.00 & -42 \\
\hline iPod Nano 4GB blue & 34 & 0.29 & -5 & HP 3050 Laserjet printer & 21 & 0.81 & 7 \\
\hline iPod Nano 4GB green & 28 & 0.18 & -5 & $\begin{array}{l}\text { Linksys WRE54G } \\
\text { wireless router }\end{array}$ & 11 & 0.27 & -6 \\
\hline iPod Nano 4GB silver & 32 & 0.28 & 0 & \begin{tabular}{|l} 
Netgear WGR614 \\
wireless router
\end{tabular} & 10 & 0.70 & 7 \\
\hline iPod 80GB black & 22 & 0.32 & -2 & & & & \\
\hline
\end{tabular}

Notes: The table reports the number of auctions and mean percentage differences between the mean auction ending price and mean low BIN price by product in the Malmendier and Lee (2011) cross-section. Positive price differences are highlighted. Prices do not include shipping fees. Products with at least ten observations are reported. 
Figure A1: Effect of auction-BIN wording difference on overbidding rate, excluding shipping fees

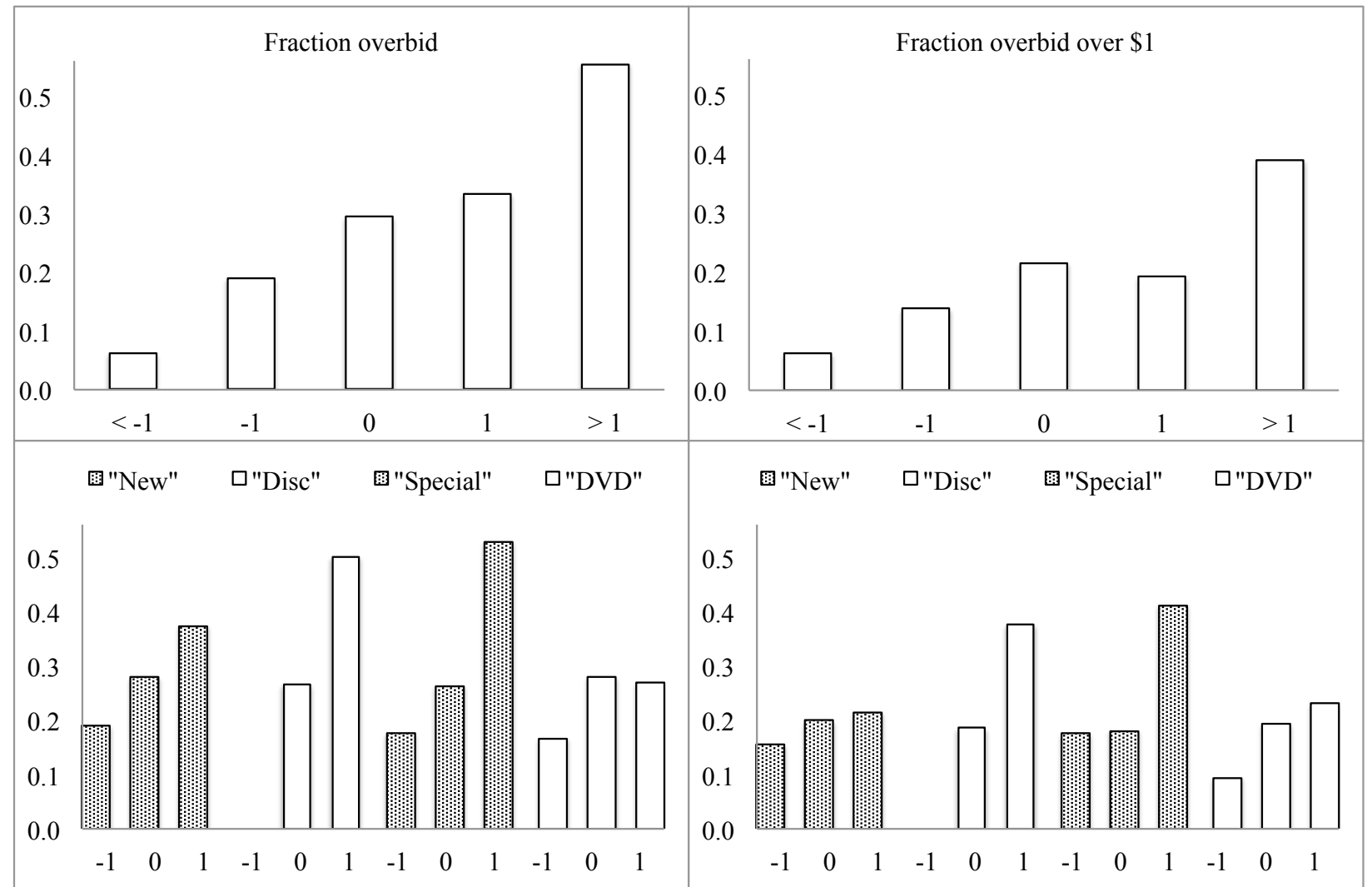

Notes: Bar heights indicate the fraction of auctions with an ending price above the low BIN price in the left panels, and above the low BIN price by over $\$ 1$ in the right panels. The top panels show these fractions according to the sum of word differences as in Section 3. The bottom panels show these fractions for individual words. Prices do not include shipping fees. 
Data
collection

\begin{tabular}{ll} 
round & Auction title \\
\hline 3 & UNLOCKED! DOLCE GABBANA v3i RAZOR! LIMITED! BID NOW!!!!
\end{tabular}

NEW OAKLEY WISDOM SNOW SNOWBOARD SKI FREE SHIP GOGGLES Canon PowerShot A630 8.0 Megapixel BRAND NEW IN BOX! Prelco Cordess Cord Electic Razor Shaver 140 XL N Philips Norelco Cordless/Cord Shaver Model \# 8140 X NEW PHILIPS NORELCO SPEEDXL MEN ELECT. RAZOR 8140XL NORELCO 8140XL

NORELCO SPEED XL CORDLESS SHAVER (BEST ON MARKET) philips norelco 8140XL recharge cordless / cord shave Philips Norelco 8140XL SpeedXL Men's Shaver

FREE INSURANCE

FREE INSURANCE

Bose LifeStyle 48 Series III

BRAND NEW MOTOROLA GOLD D\&G V3 RAZR UNLOCKED! Linksys Wireless-N Router WRT350N NOT

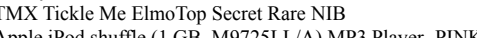

Apple iPod shuffle (1 GB, M9725LL/A) MP3 Player- PINK

Motorola V3i Dolce \& Gabbana Unlocked

NEW NOKIA N93 UNLOCKED CELLPHONE w/ EXTRAS

Quicken 2007 Basic NIB + Free Ship + Free Signo Pens

NEW Netgear WGT624 108Mbps Wireless Firewall Router

APPLE IPOD NANO (4GB 1000 SONGS) SILVER - BRAND NEW-

Brand New In Box Unlocked Dolce \& Gabanna RAZR V3i DG

NEW ETERNITY COLOGNE FOR MEN By Calvin Klein 3.4oz EDT

NETGEAR WGT624 G 108 Mbps WIRELESS ROUTER USB ADAPTER NETGEAR WGT624 $\mathrm{G}$ 108 Mbps WIRELESS ROUTER USB ADAPTE

BRAND NEW INTUIT QUICKEN DELUXE 2007 W/ TURBO TAX

Apple Ipod Nano Silver 2Gen 4GB NEW/SEALED CHEAP SHIP!

TOROLA 3 GOLD RAZR GSM PHONE

NEW Apple iPod Nano Silver 4GB/Nike + iPod Sports Kit

MTrcedes-Benz Apple iPod Nano 4GB Silver NIB New MP3

New Unlocked Gold Dolce and Gabbana Motorola Razr

Mercedes-Benz Apple iPod Nano 4GB Silver NIB New MP3

GARMIN STREETPILOT c330 AUTOMOTIVE GPS NEW In Box V8

MOTOROLA RAZR V3i D\&G \$110.00 IN EXTRAS NIB

NEW Omron HJ-12 Digtal PremiumPedometer Gift Wrapped

NEW Western Digital 500gb My Book Essential Edition $\sim \mathrm{NR} \sim$

Western Digital 500GB My Book External USB 2 Hard Drive GTarmin StreetPilot c320 GPS Newest V8 MapTalking+1GB SD Lovely Sarah Jessica Parker EDP 3.4 SEALED + bonus

BRAND NEW UNLOCKED MOTOROLA E 33 GOT W TURBO TAX

Me Mlmo

NIB Microsoft Zune ${ }^{a}$ Black (30 GB) MP3 NO RESERVE

Quicken basic 2007 NIB FREE SHIPPING

Quicken Basic 2007 Edition (Less than 5 minutes of use)

TMX Elmo Top Secret 10 th A) Digital Media Player (....
BIN title

Y BOARD GAME HERE \& NOW EDITION NEW IN BOX!!

MONOPOLY HERE \& NOW *BRAND NEW SEALED IN BOX*

New Canon PowerShot A630 8.0 MP Digital Camera w/ Card

Norelco Shaver Retainer Plate Smart Touch \& Speed XL

Norelco Philishave Charging Stand for Speed-XL \& Q Quad

Norelco Shaver Retainer Plate Smart Touch \& Speed XL

Norelco Shaver Retainer Plate Smart Touch \& Speed XL

Norelco Shaver Retainer Plate Smart Touch \& Speed XL

Norter Shaver Retiner Plate Smart Touch \& Speed XL

Norelco Shar Retiner Ple Smant Touch \& Speed XL

8 oz. Bumble and Bumble Tonic Lotion. NEW.

You on a Diet by Craig Wynett, Lisa Oz, Mehmet C. Oz...

You on a Diet by Craig Wynett, Lisa Oz, Mehmet C. Oz...

Bose Lifestyle 28 Series III BLACK New in Factory Box!

Linksys WRT300N Wireless-N Broadband Router BRAND NEW

NEW! TMX Tickle Me ELMO 10th Anniversary Sesame Stree

NEW MOTOROLA RAZR V3i GOLD D\&G DOLCE \& GABBANA MP3 V3

Nokia N90 Carl Zeis Optic New Unlocked cheaper N93 N80

Brand New NETG basic 2005 half price of $2006 \& 2007$

Brand New NETGEAR wireless PCI adapter

Apple iPod nano 2nd Gen. 2GB (MA477LL/A) silver- NEW

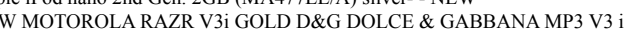

CALVIN KLEIN CK Eternity Men $3.4 \mathrm{oz}$ Cologne TESTER *NIB

Brand New NETGEAR wireless PCI adapter WG311T

Intuit Quicken Deluxe 2007 FREE SHIPPING

*** Sidekick 3 GSM T-Mobile Bluetooth ** NEW IN BOX **

Apple iPod nano 2nd Gen. 2GB (MA477LL/A) silver- - NEW

(

Apple iPod nano, 4GB, SILVER MP3 Player, NEW IN BOX

NEW! TMX Tickle Me ELMO 10th Anniversary Sesame Stree

NEW! TMX Tickle Me ELMO 10 th Anniversary Sesame Stree

NEW MOTOROLA RAZR V3i GOLD D\&G DOLCE \& GABBANA MP3 V3

Apple Ipod Nano (4GB), Silver MP3 Player
Intuit Quicken Deluxe 2007 FREE SHIPPIN

GARMIN STREETPILOT c 320 GPS Auto Car Navigation w/ V8 *NEW* MOTOROLA V3i RAZR PHONE D\&G DOLCE \& GABBANA GOLD Omron HJ-112 Premium Digital Pedometer (Ne

Western Digital 500GB My Book External USB 2 Hard Drive

GARMIN STREETPILOT c320 GPS Auto Car Navigation w/ V8

CALVIN KLEIN CK Eternity Men 3.4 oz Cologne TESTER *NIB

Lovely Perfume SARAH JESSICA PARKER 3.4 EDP Women NIB Intuit Quicken Deluxe 2007 FREE SHIPPING

*TMX* TICKLE ME FLMO 10TH ANIV EDITION Rare

LANCOME FATALE MASCARA BLACK FULL SIZE. BR AND NEW.

Brand New in box Microsoft Zunea White (30 GB)

NIB intuit Quicken basic 2005 half price of 2006\& 2007

Quicken 2007 Basic NIP Intuit Free Shipping!

Brand New in box Microsoft Zune ${ }^{a}$ White ( $30 \mathrm{~GB}$ ) \begin{tabular}{ccc} 
Auction price & Bin price & Explanation \\
\hline 209.5 & 20.2 Different products
\end{tabular}

9.99 Auction includes "Mem card"

23 Different products

40.45 BIN includes "Card"

17.75 Different products

14.99 Different products

17.75 Different products

7.75 Different products

17.75 Different products

17.75 Different products

17.75 Different products

24.44 Auction appears to have 5 bottle

5.49 Different products

184.49 Different product

159 BIN includes "accesories"

110 Auction is "NOT wrt300n"

81.99 Different identifying numbers

159 BIN has "accessories"

169.99 Auction is unlocked

397.99 Different products

18.98 Auction has "Free Signo Pens" and BIN is 2005 editio

32.98 Different products

136.95 Auction iscludes "Toner"

169.99 Auction is unlocked

$38.87 \mathrm{BIN}$ is tester

37.98 Different product

37.98 Different products
29.99 Auction includes Tu

355 Auction includes Turbo Tax

36.95 Auction is $4 \mathrm{~GB}$, BIN is $2 \mathrm{~GB}$

$149.5 \mathrm{BIN}$ in unbranded version

185 Auction has Nike Sports Ki

136.95 Auction is 4GB, BIN is $2 \mathrm{~GB}$

(v.5 Auction has the Warning Box (variation)

2.5 Auction has he Top Secret Box (variation)

的.99 Auction includes Turbo Tax editio

222.98 Different products

214.98 Auction has extras

20.94 Auction is gift wrapped

170.94 "Essential Edition" is different type of hard drive

different type of hard drive

22.98 Auction has
38.87 BIN is tester

29.99 Auction has "bonus"

35 Auction has the Top Secret Box (variation)

13.94 Auction is unboxe

$8.98 \mathrm{BIN}$ in 2005 versi

24 Auction is used 
Microsoft Zunea Black (30 GB) Digital Media Player BRAND NEW UNLOCKED MOTOROLA V3 GOLD RAZR GSM PHONE NEW B ML ELM TMX TOP SECRET NEW BOXED SEALED NIB! West 30 Digs Cordess Shaver Razor 8000 w/ Extras Wester Dint 500 B My B Brand New Pink Apple Ipod Nano 4GB with Warranty Lovely Sarah Jessica Parker $3.4 \mathrm{oz}$ * NIB * perfume *edp LINKSYS WIRELESS-G RANGE EXPANDER WRE54G - ALMOST NEW Canon PowerShot SD900 Digital ELPH Coach Edition Gif...

WESTERN DIGITAL MY BOOK 500GB EXTERNAL HARD DRIVE BRAND NEW UNLOCKED MOTOROLA V3 GOLD RAZR GSM PHONE RAZR V3i - Dolce and Gabbana Special Edition N.R.

BRAND NEW APPLE IPOD NANO 4GB BLUE GEN 2 WITH WARRANTY ETERNITY 3.4 CALVIN KLEIN PERFUME WOMEN CK RETAIL BOX Apple iPod ${ }^{a}$ shuffle 2nd Gen Orange 1 GB, MA954LL/A.-NEW

BRAND NEW UNLOCKED MOTOROLA 3 G RAND NEW UNLOCKED MOTOROLA V3 GOLD RAZR GSM PHON NEW APPLE 2ND GEN 4GB PINK IPOD NANO \& CASES NO RESERVE TICKLE ME ELMO TMX TOP SECRET $\sim$ BNIB $\sim$ ENGLISH
NEW XBOX 360 WIRELESS CONTROLLER SEALED BLACK

NEW MOTOROLA V3I RAZR BLACK \& GOLD UNLOCKED LIMITED DLACK XBOX 360 ELTE WIRELESS GOLTDOLLOR *BEANIMIED WD Western Digital My Book 500GB External USB drive

NEW AUTHENTIC ETERNITY - CALVIN KLEIN 3.40z EDP PERFUME LOVELY SARAH JESSICA PARKER 3.4 oz EDP NIB NO RESERVE NEW AUTHENTIC ETERNITY - CALVIN KLEIN 3.40Z EDP PERFUME Western Digital 500GB My Book External USB 2 Hard Drive

Western Digital 500GB Ry Botal E

Zune 30GB Digital Media MP3 Player Black NEW -

BRAND NEW MICROSOFT XBOX 360 WIRELESS CONTROLLER X BOX BRAND NEW UNLOCKED MOTOROLA V3 GOLD RAZR GSM PHONE ETERNITY by CALVIN KLEIN 3.4 FL. OZ. FOR WOMEN NIB

Brand New Pink Apple Ipod Nano 4GB with Warranty

BRAND NEW UNLOCKED MOTOROLA V3 GOLD RAZR GSM PHONE **NEW** IN BOX SIDEKICK 3

Omron HJ-112 Pedometer - Like New - Used Once

作

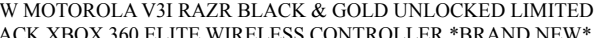

Oral B Triumph 9100 Professional Toothbrush 9400 / 9450

IGB Apple iPod Shuffle Pink

Apple iPod Shuffle Green 1 GB NEW SEALED MA951LL/A

ETERNITY by Calvin Klein 3.4 oz EDP perfume *SEALED*

TEXAS INSTRUMENTS TI-89 TITANIU SEALED BOX W / UPC Apple iPod Shuffle Blue 1 GB BRAND NEW SEALED MA949LL/A

XBOX 360 WIRELESS CONTROLLR
GARMIN $\$ 350$ STREETPILOT c320 GPS BRAND NEW, V8 MAPS, NR Lovely 3.4 EDP spray by Sarah Jessica Parker Tester nib

Apple Ipod Nano Mercedes-Benz Edition (4GB), Silver NEW 1 GB Apple iPod Shuffle Pink

*T-Mobile Sidekick 3 (T-Mobile) Brand New - No Reserve* **Brand New** T-Mobile Sidekick 3 w/car charger!!!
Brand New in box Microsoft Zune ${ }^{a}$ White ( $\left.30 \mathrm{~GB}\right)$

( B.W. TMX Tickle Me ELMO 10th Anniversary Sesame Street Western Dicita $\mathrm{My}$ cook Essentin 500

My Book Essential 500gb

New Pink Ipod Nano 4GB- Factory Sealed -Free Shipping

Lovely* Sarah J Gold) Unlocked EDP Women TST NIB

Linksys Wireless G Range Expander 2.4GHz WRE54C

Canon PowerShot SD900 Digital ELPH 10 Megapixel ${ }^{* *}$ NEW $^{* *}$

Brand New NETGEAR wireless PCl adapte
Western Digital My Book Essential $500 \mathrm{gb}$

branded New Rare

NEW MOTOROLA V3i GOLD RAZR UNLOCKED MP3 BLUETOOTH PHONE

Apple" 4GB iPod" Nano (Blue) New in Factory Sealed Box

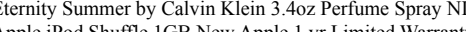

Motorad Razr V3 (Gold) Un Apple 1 yr Limited Warranty

Motorola Raz V3 (Gold) Unlocked Unbranded New Rare

APPLE IPOD NANO (2ND GEN) 4Gb. HOT PINK ' NEW IN BOX

NEW 600 CON TROLLER JOYTECH SE ADVANCED BRAND NEW

X

Western Digital My Book Essential 500gb

Eternity Summer by Calvin Klein 3.40z Perfume Spray NIB

Lovely * Sarah Jessica Parker 3.4 EDP Women TST NIB

Wernity Summer by Calvin Klein 3.40z Perfume Spray NIB

Western Digital My Book Essential 500gb

3 3 BLUETOOTH PHONE

Western Digital My Book Essential 500gb

Western Digital 500GB My Book External USB 2 Hard Drive

Brand New in box Microsoft Zune ${ }^{a}$ White $(30 \mathrm{~GB})$

XOX 360 CONTROLLER JOYTECH SE ADVANCED BRAND NEW

Eternity Summer by Calvin Klein $3.40 z$ Perfume Spw Rare

Motorola Razr V3 ( Gold) Unlocked Unbranded New Rare

Apple Ipod Nano (4GB), Pink- Brand new, Factory Sealed

Western Digital My Book Essential 500gb

T-Mobile Sidekick ID (1-Mobile) Brand Ne

Ipod Shuffle I GB Blue 2nd Second Gen NEW in box+EXTRAS

WW OMRN HJ-112 HJH2 WALKING PEDOMETER

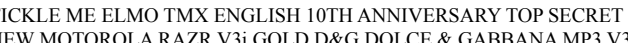
XBOX 360 CONTROLLER JOYTECH SE ADVANCED BRAND N WW NEW - Oral-B Triumph 9400 Power Toothbrush

Apple iPod Shuffle 1GB New Apple 1 yr Limited Warranty
NEW Apple iPod Shuffle 1GB 2nd GEN MA952LL/A GREE

NEW Apple iPod Shuffle 1GB 2nd GEN MA952LL/A GREIN

Calvin Klein Eternity Men $3.4 \mathrm{oz}^{*}{ }^{*} \mathrm{NIB} * 100 \%$ Authentic

QUICKEN 2007 BASIC PERSONAL FINANCES-NEW UNREGISTERED!!

Texas Instruments TL-89 Titanium Scientific Calculator

NEW Apple iPod Shuffle 1GB 2nd GEN MA950LL/A BLUE

X BOX 360 CONTROLLER JOYTECH SE ADVANCED BRAND NEW

Brand New In The Box!!!!! Garmin c330 Priced To Sell

ETERNTY by Cali Kl i 3.4 COLOGNE SP MEN NEW NN BOX

Apple iPod Shuffle 1GB New Apple 1 yr Limited Warranty

SEALED BRAND NEW 4GB SILVER APPLE IPOD NANO 4 GB MP3

BRAND NEW TMOBILE SIDEKICK 3 UNLOCKED!!! FOR ALL GSM

Calvin Klein Etenity Meb 34 Butooth
210 Different color

(variation)

170 "Essential Edition" is different type of hard drive

170 "Essential Edition" is different type of hard drive

175 Auction has Warranty

$6.99 \mathrm{BIN}$ is tester

315 Auction is used

170 "Essential Edition" is different type of hard drive

$149.5 \mathrm{BIN}$ is unbranded version

4.95. Auction has warranty

77 BN has warranty

$49.5 \mathrm{BIN}$ is unbranded version

149.5 BIN is unbranded versio

181.99 Auction has cases

40 Auction has the Top Secret Box (variation)

43.66 Different brands

169.99 Auction is unlock

170 "Essential Edition" is different type of hard drive

24 Auction missing UPC (for rebate)

$39.8 \mathrm{BIN}$ is summer

$36.99 \mathrm{BIN}$ is tester

$43.8 \mathrm{BIN}$ is summer

170 "Essential Edition" is different type of hard drive
215.89 BIN is unlocked

170 "Essential Editio

is different type of hard drive

210 Different colors

43.66 Different brands

149.5 BIN is unbranded version

$43.8 \mathrm{BIN}$ is summer

182.99 Auction has warranty

170 "Essential Edition" is different type of hard drive

330 Sidekick 3 vs Sidekick ID

77.98 BIN has extras
22.44 Auction is used

40 Auction has Warning Box, BIN has the Top Secret Box (variation)

169.99 Auction is unlocked

43.66 Different brands

66.949100 vs 9400

77 BIN has warranty

81.99 Different ID numbers

38.98 Auction is perfume, $\mathrm{BIN}$ is cologne

104.98 Auction is opened

81.99 Differnet ID number

43.66 Different brands

275 Different products
32.44 Auction is tester

41.98 Auction is perfume, BIN is cologne

77 BIN has warranty

182.49 Auction is Mercedes-Benz edition

77 BIN has warranty
384.98 BNN is unlocked

$384.98 \mathrm{BIN}$ is unlocked

355 Auction has car char 
ORANGE Apple 1GB 2nd Generation iPod Shuffle

Bumble and Bumble Tonic Lotion, 8 ounce spray no box

NOKIA N93 GSM TRIBAND PHONE, UNLOCKED, NEW IN BOX!!!

XBOX 360 wheless controller nhw unopened

Western Digital My Book Essential 500gb

Sidekick iD T-Mobile NIB \& extra Dark Gray Bumper!

New In Box Sidekick 3 with extra battery

brand new side kick 3

T-Mobile Sidekick ID (T-Mobile)

Conce spray no bo

NEW D-LINK WIRELESS G ROUTER DI-524 802.11g AIRPLUS

T-Mobile Sidekick ID (T-Mobile)

NEW Canon SD600 PowerShot Digital Camera SD 600

Callaway HX HOT NEW golf balls 6 Dozen

USED BELKIN WIRELESS G ROUTER 802.11

(1)

NEW Western Digital 500G My Book USB HBS CAv 500 GIGATION V8

Murad Acne Spot Treatment acne/oily skin -NIB-
Apple iPod Shuffle 1GB New Apple 1 yr Limited Warranty

NEW NOKIA :N93 3.2 MPIXEL VIDEO GSM VIDEOCAM PHONE+WIF

$X$ BOX 360 CONTROLLER JOYTECH SE ADVANCED BRAND NEW

Brand New is 500 GB My Book External USB 2 Hard Drive

NEW T-Mobile Sidekick 3 III PDA GSM Bluetooth NIB

BRAND NEW T-Mobile Sidekick 3 (T-Mobile) FREE SHIPPING

T-Mobile Sidekick ID (T-Mobile) Brand New

BRAND NEW T-Moble Sidekick 3 (T-Mobile) FREE SHIPPING

oz. Bumble and Bunble Tonic Lotion. NEW.

Megapixel**NEW**

SIDEKICK 3 PHONE BRAND NEW IN A BOX SWEET CAMERA PHONE!

CALLAWAY HX TOUR GOLF BALLS - BRAND NEW!!! - 6 DOZEN

Belkin (F5D7230-4) 54Mbps Wireless G Router

NEW Western Digital $500 \mathrm{gb}$ My Book Essential Edition NR

MURAD TV ACNE COMPLEX KIT - Large Sizes NEW in box
77 BIN has warranty

24.44 Auction has no box

650.99 Auction is unlocked

43.66 Different brands

170.94 "Essential Edition" is different type of hard drive

275 Different products
365 Sidekick 3 vs Sidekick ID, and auction has bumber

350 Auction has extra batter

330 Sidekick 3 vs Sidekick ID

350 Sidekick 3 vs Sidekick

319 Different products

37.75 Different routers

334.98 Sidekick 3 vs Sidekick ID

324.99 Different products

210 Different types of golf balls (Hot vs Tour)

558. 95 Auction is used

522.48 Different products

156.94 "Essential Edition" is different type of hard drive

47.9 Different sizes/products 


\begin{tabular}{|c|c|c|c|c|}
\hline \multirow[t]{2}{*}{ 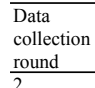 } & & & \multicolumn{2}{|c|}{ Auction price Bin price $\quad$ Explanation } \\
\hline & Canon PowerShot A630 8.0 Megapixel+Mem card & Canon PowerShot A630 8.0 Megapixel Digital Camera NEW & 181.00 & 0.99 Auction includes "Mem card" \\
\hline 3 & UNLOCKED! DOLCE GABBANA v3i RAZOR! LIMITED! BID NOW!!!! & & 170.50 & 9.65 Different products \\
\hline 2 & NEW OAKLEY WISDOM SNOW SNOWBOARD SKI FREE SHIP GOGGLES & NEW 2006 MONOPOLY HERE AND NOW BOARD GAME SEALED & 121.00 & 14.99 Different products \\
\hline 2 & Norelco 8140 XL Rechargeable Shaver-NEW in BOX & Norelco Philishave Charging Stand for Speed-XL \& Quadra & 49.00 & 9.99 Different products \\
\hline & Norelco Cordless Cord Electric Razor Shaver 8140XL New & Norelco Shaver Retainer Plate Smart Touch \& Speed XL & 66.55 & 14.50 Different products \\
\hline 2 & Philips Norelco Cordless/Cord Shaver Model \# 8140 XL & Norelco Shaver Retainer Plate Smart Touch \& Speed XL & 64.00 & 14.50 Different products \\
\hline 3 & NORELCO SPEED XL CORDLESS SHAVER (BEST ON MARKET) & Norelco Shaver Retainer Plate Smart Touch \& Speed XL & 52.26 & 14.50 Different products \\
\hline 3 & NEW PHILIPS NORELCO SPEEDXL MEN ELECT. RAZOR 8140XL & Norelco Shaver Retainer Plate Smart Touch \& Speed XL & 52.00 & 14.50 Different products \\
\hline 2 & NORELCO $8140 \mathrm{XL}$ & Norelco Shaver Retainer Plate Smart Touch \& Speed XL & 50.00 & 14.50 Different products \\
\hline 3 & PHILIPS NORELCO SPEED XL CORDLESS RAZOR 8140 XL 8140XL & Norelco Shaver Retainer Plate Smart Touch \& Speed XL & 47.10 & 14.50 Different products \\
\hline 3 & Philips Norelco 8140XL SpeedXL Men's Shaver & Norelco Shaver Retainer Plate Smart Touch \& Speed XL & 45.00 & 14.50 Different products \\
\hline 2 & philips norelco 8140XL recharge cordless / cord shaver & Norelco Shaver Retainer Plate Smart Touch \& Speed XL & 45.00 & 14.50 Different products \\
\hline 1 & FREE INSURANCE & You on a Diet by Craig Wynett, Lisa Oz, Mehmet C. Oz... & 30.00 & 9.99 Different products \\
\hline 1 & FREE INSURANCE & You on a Diet by Craig Wynett, Lisa Oz BRAND NEW \$18.25 & 28.99 & 9.99 Different products \\
\hline 3 & New $\sim 5 \sim 8$ oz. Bumble and bumble Hair Tonic & 8 oz. Bumble and Bumble Tonic Lotion. NEW. & 50.00 & 17.49 Auction inclues five 8 ounce containers \\
\hline 2 & New Oral B S-320 Sonic Complete Rechargeable Toothbrush & ORAL B BRAUN VITALITY Sonic Toothbrush Shipping DEAL & 24.50 & 8.95 Different products \\
\hline 3 & BOSE Lifestyle 48 Series III Home Theater System Black & NEW BOSE LIFESTYLE 38 SERIES III BLACK FACTORY SEALED & 5100.00 & 2299.00 Different products \\
\hline 3 & 'NIB" BOSE LIFESTYLE 48 SERIES III HOME ENTERTAINMENT & Bose Lifestyle 28 Series III BLACK BRAND NEW NOT REFURB & 3282.32 & 1500.00 Different products \\
\hline 3 & NEW unopened Garmin StreetPilot $\mathbb{B}$ c 550 GPS NO RESERVE!!! & GARMIN STREETPILOT c 320 GPS Auto Car Navigation w/ V8 & 405.02 & 204.99 Different products \\
\hline 2 & MICROSOFT XBOX 360 WIRELESS CONTROLLER - FREE SHIPPING & X BOX 360 CONTROLLER JOYTECH SE ADVANCED BRAND NEW & 38.00 & 20.04 Different brands \\
\hline 2 & Omron HJ-112 Pedometer - Like New - Used Once & NEW IN BOX Omron HJ-112 Digital Premium Pedometer HJ112 & 16.74 & 8.98 Auction is used \\
\hline 3 & BLACK XBOX 360 ELITE WIRELESS CONTROLLER *BRAND NEW* & X BOX 360 CONTROLLER JOYTECH SE ADVANCED BRAND NEW & 37.00 & 19.85 Different brands \\
\hline 2 & You the owner's manual \& You on a diet,NEW,combo,hb,dj! & You on a Diet by Mehmet C. Oz, Mehmet Oz M.D., Micha... & 17.65 & 9.63 Auction has two books \\
\hline 2 & New in box Xbox 360 Wireless Controller & $\mathrm{X}$ BOX 360 CONTROLLER JOYTECH SE ADVANCED BRAND NEW & 36.00 & 20.04 Different brands \\
\hline 3 & XBOX360 BLACK WIRELESS CONTROLLER NEW \& FACTORY SEALED & $\mathrm{X}$ BOX 360 CONTROLLER JOYTECH SE ADVANCED BRAND NEW & 33.99 & 19.85 Different brands \\
\hline 3 & Quicken 2007 Basic NIB + Free Ship + Free Signo Pens & NIB intuit Quicken basic 2005 half price of $2006 \& 2007$ & 25.51 & $14.99 \mathrm{BIN}$ is 2005 version \\
\hline 3 & Bose LifeStyle 48 Series III & Bose Lifestyle 28 Series III BLACK New in Factory Box! & 3050.00 & 1800.00 Different products \\
\hline 3 & NEW XBOX 360 WIRELESS CONTROLLER SEALED BLACK & $\mathrm{X}$ BOX 360 CONTROLLER JOYTECH SE ADVANCED BRAND NEW & 33.50 & 19.85 Different brands \\
\hline & NEW Omron HJ-112 Digital PremiumPedometer Gift Wrapped & NEW IN BOX Omron HJ-112 Digital Premium Pedometer HJ112 & 14.99 & 8.98 Auction is "gift wrapped" \\
\hline 3 & BLACK XBOX 360 ELITE WIRELESS CONTROLLER *BRAND NEW* & X BOX 360 CONTROLLER JOYTECH SE ADVANCED BRAND NEW & 33.02 & 19.85 Different brands \\
\hline 2 & XBOX 360 WIRELESS CONTROLLER NEW!! & X BOX 360 CONTROLLER JOYTECH SE ADVANCED BRAND NEW & 33.00 & 20.04 Different brands \\
\hline 3 & 30Gb Black Zune with Travel Pack \& Car Kit & MICROSOFT ZUNE mp3 video player $30 \mathrm{~GB}$ & 275.00 & 170.00 Auction includes "Travel Pack and Car Kit" \\
\hline 1 & BRAND NEW MOTOROLA GOLD D\&G V3 RAZR UNLOCKED!! & Motorola razr V3 new unlocked gold edition, accesories & 232.50 & 148.00 BIN includes "accessories" \\
\hline 3 & BRAND NEW MICROSOFT XBOX 360 WIRELESS CONTROLLER X BOX & X BOX 360 CONTROLLER JOYTECH SE ADVANCED BRAND NEW & 31.00 & 19.85 Different brands \\
\hline 2 & Sidekick 3 & new sidelick3 Tmobile phone music player with WARRANTY & 495.00 & 325.00 BIN includes "WARRANTY" \\
\hline & XBOX 360 wireless controller new unopened & X BOX 360 CONTROLLER JOYTECH SE ADVANCED BRAND NEW & 29.50 & 19.85 Different brands \\
\hline $\begin{array}{l}1 \\
2\end{array}$ & Apple iPod shuffle (1 GB, M9725LL/A) MP3 Player- PINK & NEW Apple iPod Shuffle 1GB 2nd GEN MA948LL/A PINK & 102.50 & 70.99 Different item numbers \\
\hline 1 & NIB HP LaserJet 3050 All in One Printer w/ Toner New & NEW HP LaserJJet 3050 All-In-One Laser Printer Scan Fax & 199.50 & 139.99 Auctions includes "Toner" \\
\hline $\begin{array}{l}1 \\
2\end{array}$ & Linksys Wireless-N Router WRT350N NOT wrt300n **NEW** & Linksys WRT300N Wireless-N Broadband Router BRAND NEW & $\begin{array}{l}150.00 \\
5500\end{array}$ & 108.00 Auction is "NOT wrt300n" \\
\hline & $\begin{array}{l}\text { NEWNOKOKA N93 UNLOCKED CELLPHONE WE EXTRAS } \\
\text { ETERNITY by Calvin Klein } 3.4 \text { oz edp Perfume SEALED NIB }\end{array}$ & $\begin{array}{l}\text { Nokia N90 Carl Zeis Optic New Unlocked cheaper N93 N80 } \\
\text { Calvin Klein Eternity Men } 3.407 \text { *NI* } 100 \% \text { Authentic }\end{array}$ & $\begin{array}{r}525.00 \\
39.95\end{array}$ & $\begin{array}{l}380.00 \text { Different products } \\
28.99 \text { Auction is for women ("perfume"). BIN is for men }\end{array}$ \\
\hline & $\begin{array}{l}\text { XBOX } 360 \text { WIRELESS CONTROLLER } \\
\text { X }\end{array}$ & X BOX 360 CONTROLLER JOYTECH SE ADVANCED BRAND NEW & 26.99 & 19.85 Different brands \\
\hline & Quicken basic 2007 NIB FREE SHIPPING & NIB intuit Quicken basic 2005 half price of $2006 \& 2007$ & 20.29 & 14.99 BIN in 2005 version \\
\hline & APPLE IPOD NANO (4GB 1000 SONGS) SILVER - BRAND NEW- & Apple iPod nano 2nd Gen. 2GB (MA477LL/A) silver- - NEW & 171.50 & $127.004 \mathrm{~GB}$ vs $2 \mathrm{~GB}$ \\
\hline 1 & NEW ETERNITY COLOGNE FOR MEN By Calvin Klein 3.40z EDT & CALVIN KLEIN CK Eternity Men 3.4 oz Cologne TESTER *NIB & 38.99 & 28.88 Auction is tester \\
\hline & Gold Motorola V3 Razr Cell Phone Unlocked Cellphone & Motorola razr V3 new unlocked gold edition, accesories & 198.99 & 148.00 Auction is unlocked and BIN includes accessories \\
\hline & LOST SEASON ONE 1 DVD BOX SET NEW SEALED COMPLETE SET & Scrubs: The Complete Fourth Season (2006, DVD) & 26.00 & 19.99 Different products \\
\hline 3 & Motorola V3i Dolce \& Gabbana Unlocked & NEW MOTOROLA RAZR V3i GOLD D\&G DOLCE \& GABBANA MP3 V3 i & 217.50 & 169.99 Auction is unlocked \\
\hline 3 & Brand New T-Mobile Sidekick 3 Unlocked GSM MP3 Phone & *** Sidekick 3 GSM T-Mobile Bluetooth ** NEW IN BOX ** & 415.00 & 330.00 Auction is unlocked \\
\hline 3 & Apple Ipod Nano Silver 2Gen 4GB NEW/SEALED CHEAP SHIP! & Apple iPod nano 2nd Gen. 2GB (MA477LL/A) silver- - NEW & 159.49 & $127.004 \mathrm{~GB}$ vs $2 \mathrm{~GB}$ \\
\hline 2 & LOVELY SARAH JESSICA PARKER 3.4 oz EDP NIB & LOVELY by SARAH JESSICA PARKER 3.4 OZ EDP WOMAN [UNBOX] & 35.00 & 27.99 BIN is unboxed \\
\hline 1 & ETERNITY by Calvin Klein 3.4 oz. EDT Cologne men NEW NR & CALVIN KLEIN CK Eternity Men 3.4 oz Cologne TESTER *NIB & 36.00 & 28.88 BIN is tester \\
\hline & Bose® Lifestyle® 48 Series III & NEW BOSE LIFESTYLE 38 SERIES III BLACK FACTORY SEALED & 2850.00 & 2299.00 Different products \\
\hline 3 & New Xbox 360 Wireless Controller NIB NR & X BOX 360 CONTROLLER JOYTECH SE ADVANCED BRAND NEW & 24.50 & 19.85 Different brands \\
\hline 1 & BRAND NEW UNLOCKED MOTOROLA V3 GOLD RAZR GSM PHONE & Motorola Razr V3 ( Gold) Unlocked Unbranded New Rare & 162.50 & $132.49 \mathrm{BIN}$ is unbranded version \\
\hline 2 & GARMIN STREETPILOT c330 AUTOMOTIVE GPS NEW In Box V8 & NEW Garmin StreetPilot c320 GPS Receiver Car Navigation & 244.02 & 199.00 Different products \\
\hline 1 & NEW Apple iPod Nano Silver 4GB/Nike + iPod Sports Kit & Apple iPod nano, 4GB, SILVER MP3 Player, NEW IN BOX & 212.50 & 175.00 Auction has Nike + iPod Sports Kit \\
\hline 1 & TMX Tickle Me ElmoTop Secret Rare NIB & New* TMX ELMO Tickle Me Elmo T.M.X. Sealed English Ver. & 39.99 & 32.99 Auction has the Top Secret Box (variation) \\
\hline 1 & Tickle Me ELMO TMX 10th ANNIVERSARY TOP SECRET NEW NIB & New* TMX ELMO Tickle Me Elmo T.M.X. Sealed English Ver. & 39.99 & 32.99 Auction has the Top Secret Box (variation) \\
\hline 3 & Mercedes-Benz Apple iPod Nano 4GB Silver NIB New MP3 & Apple iPod nano 2nd Gen. 2GB (MA477LL/A) silver- - NEW & 150.49 & 127.00 Auction is Mercedes version \\
\hline 3 & NEW! Canon PowerShot A630 8.0 Megapixel w/ Warranty! & Canon PowerShot A630 8.0 Megapixel DIGITAL Camera & 232.50 & 199.00 Auction has warranty \\
\hline 1 & NEW Western Digital 500gb My Book Essential Edition $-\mathrm{NR} \sim$ & WD Western Digital My Book 500GB External USB drive & 180.00 & 154.99 "Essential Edition" is different type of hard drive \\
\hline 2 & Tickle Me Elmo - TMX - Brand New in Top Secret Box!!! & TMX Tickle Me Elmo English Version Anniv. Ed. *NIB* & 29.00 & 24.99 Auction has the Top Secret Box (variation) \\
\hline & Quicken 2007 Basic, NIB Sealed, UPC intact \$0 Shipping & 2007 QUICKEN Basic Personal Finance Software New In Box & 23.01 & 19.99 Auction includes UPC (for rebate) \\
\hline
\end{tabular}


NEW HP LaserJet 3050 AIO Printer/Copy/ScanFax Laser Jet
MOTOROLA RAZR V3i D\&G \$110.00 IN EXTRAS NIB

BRAND NEW UNLOCKED MOTOROLA V 3 GOLD RAZR GSS Western Digtal

TICKLE ME ELMO WARNING TMX 10TH ANNIVERSARY ENGLISH Lovely Sarah Jessica Parker EDP 3.4 SEALED + bonus

Lost - The Complete Season 1 DVD Set NEW

Garmin StreetPli

Mercedes-Benz Apple iPod Nano 4GB Silver NIB New MP3

Lancome FATALE Mascara NOIR/Black New unboxed

BRAND NEW HP LaserJet 3050 All-in-One Laser Jet Printer

INKSYS WIRELESS-G RANGE EXPANDER WRE54G - ALMOST NEW

(

New Unlocked Gold Dolce and Gabbana Motorola Razr
BRAND NEW APPLE IPOD NANO 4GB BLUE GEN 2 WITH WARRANTY New Unlocked Motorola V3 Gold Razr GSM Phon

Apple iPod ${ }^{\mathrm{TM}}$ shuffle 2 2nd Gen Orange 1 GB, MA954LL/A.-NEW $1 \mathrm{~GB}$ Apple iPod Shuffle Pink

Microsoft Zune TM Black (30 GB) Digital Media Played (...

BRAND NEW UNLOCKED MOTOROLA V3 GOLD RAZR GSM PHONE Quicken 2007 Basic Personal Finances SEALED!

(3) 3.4 D.EDT PERFUME WOMEN Sea

BRAND NEW UNLOCKED MOTOROLAX - SEALD

Quicken Basic 2007 Edition (Less than 5 minutes of use)

New sealed Quicken Basic 2007 NIB w/ UPC

NEW MOTOROLA 31 RAZR D\&G DOLCE \& GABBANA GOLD

TY by Calvin Klein 3.4 oz. EDT PERFUME WOMEN Sea

Brand New Pink Apple Ipod Nano 4GB with Warranty

Brand New Pink Apple Ipod Nano 4GB with Warranty
NIB Microsoft Zune ${ }^{\mathrm{TM}}$ Black (30 GB) MP3 NO RESERVE!!

New Callaway HX Tour 5620076 Dozen Golf Balls No Log

NEW Tickle Me Elmo TMX TOP SECRET Unopened Box ENGLISH

Tickle Me Elmo - TMX - Brand New in Top Secret Box!!!

Nokia N93 featuring a 3.2 megapixel camera

TICKLE ME ELMO TMX TOP SECEET BNIB ENGLISH

NEW APPLE 2ND GEN 4GB PINK IPOD NANO \& CASES NO RESERVE

Canon PowerShot SD900 Digital ELPH Coach Edition Gif...
INTUIT QUICKEN 2007 BASIC NEW IN SEALED BOX W / UPC

1GB Apple iPod Shuffle Pink

Microsoft Zune Black 30 GB MP3 Video Player SEALED BOX

ETERNITY BY CALVIN KLEIN FOR WOMEN EDP 3.4 FL.OZ BNIB

Brand New Pink Apple Ipod Nano 4GB with Warranty
BRAND NEW UNLOCKED MOTOROLA V3 GOLD RAZR GSM PHON

BRAND NEW UNLOCKED MOTOROLA V3 GOLD RAZR GSM PHON
Western Digital 500 GB Essential My Book ** Brand New**

Western Digital 500GB Essential My Book ** Brand New**
BRAND NEW UNLOCKED MOTOROLA V3 GOLD RAZR GSM PHONE

Apple iPod Shuffle Green 1 GB NEW SEALED MA951LL/A

Microsoft Zune - Black - 30 GB - New in Box!!!! No Res

ETERNITY by Calvin Klein 3.4 oz. EDT PERFUME WOMEN Seal

ERERNITY by Calvin Klein 3.4 oz. EDT PERFUME WOMEN Seal

BRAND NEW UNLOCKED MOTOROLA 3 GOLD RAZR GSM PHO

LOVELY SARAH JESSICA PARKER PERFUME 3.4 OZ SEALED

Quicken 2007 Basic sealed, without UPC
NEW BRAUN 8985360 COMPLETE ELECTRIC SHAVER RAZOR 8995 NEW BRAUN 8985360 COMPLETE ELECTRIC SHAVER RAZOR 8995 Lovely 3.4 EDP spray by Sarah Jessica Parker Tester nib

LOVELY SARAH JESSICA PARKER PERFUME 3.4 OZ SEALED

Brand new Apple ipod shuffle (blue)
ETERNITY BY CALVIN KLEIN FOR WOMEN EDP 3.4 FL.OZ BNIB
Brand New HP LaserJet 3050 AIO Printer w/ Ink + USB

列

NEW Western Digital 500gb My Book Essentil Ew Rare

列 New* TMX ELMO Tickle Me Elmo T.M.X. Sealed English Ver.

Scrubs: The Complete Fourth Season (2006, DVD)

GARMIN STRETERLOT C327 GUS Auto Car Navigation w/

Apple Ipod Nano (4GB), Silver MP3 Player
SARAH JESSICA PARKER Lovely EDP $3.4 \mathrm{oz} / 100 \mathrm{ml}$ TST NIB

LANCOME FATALE MASCARA BLACK FULL SIZE. BRAND NEW.

Brand New HP LaserJet 3050 AIO Printer w/ Ink + USB

Linksys Wireless G Range Expander 2.4GHz WRE54G

Braun electric 360 complete razor NEW

NEW MOTOROLA RAZR V3i GOLD D\&G DOLCE \& GABBANA MP3 V3

Apple $\circledast 4$ 4GB iPod $囚$ Nano (Blue) New in Factory Sealed Box

Motorola Razr $\sqrt{ }$ ( Gold) Unlocked Unbranded New Rare

Apple iPod Shuffle 1GB New Apple 1 y Ling pd Wa HN

Apple iPod Shuffle 1 GB New Shuffle 1 GB New Apple I yr Limited Warranty

NIB *HOT* Limited Edition Microsoft ZUNE pretty PINK!

Motorola Razr V3 ( Gold) Unlocked Unbranded New Rare

NIB intuit Quicken basic 2005 half price of $2006 \& 2007$
* ETERNITY PURPLE ORCHID * 3.4*by * CK * Perfume* NIB
NIB *HOT* Limited Edition Microsoft ZUNE pretty PINK!

Motorola Razr V3 ( Gold) Unlocked Unbranded New Rare

2007 QUICKEN Basic Personal Finance Software New In Box

QUICKEN 2007 BASIC NEW IN SEALED BOX

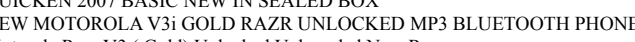

Motorola Razr

Apple Ipod Nano (4GB), Pink- Brand new, Factory Sealed

CB "HOT" Limited Edition Microsoft ZUNE pretty PINK!

CALLAWAYSIX TARK GR LOFLD BALND NEW!!! - 6 DOZEN

Tickle Me FLMO TMX ENGLISH Speaking $\sim$ BRAND NEW IN BOX

Tickle Me $\sim$ ELMO TMX $\sim$ ENGLISH Speaking $\sim$ BRAND NEW IN BOX

Nokia N90 Carl Zeis Optic New Unlocked cheaper N93 N80

The Me ELMO TMX

Quicken DELUXE delux 2007, FULL VERSION, Delivery in
APPLE IPOD NANO (2ND GEN) 4Gb. HOT PINK ' NEW IN BOX

QUICKEN 2007 BASIC PERSONAL FINANCES-NEW UNREGISTERED!

Apple iPod Shuffle 1GB New Apple 1 yr Limited Warranty

(HoT) Brand New

* ETERNITY PURPLE ORCHID * 3.4*by * CK * Perfume* NIB

Apple Ipod Nano (4GB), Pink- Brand new, Factory Sealed

Motorola Razr V3 ( Gold) Unlocked Unbranded New Rare

WD Western Digital My Book 500GB External USB driv

NEW Apple iPod Shuffle 1 GB 2nd GEN MA952LL/A RR

NIB *HOT* Limited Edition Microsoft ZUNE pretty PINK!

* ETERNITY PURPLE ORCHID * 3.4*by * CK * Perfume* N

"

Motorola Razr V3 (Gold) Unlocked Unbranded New Rare

LOVELY by SARAH JESSICA PARKER 3.4 OZ EDP WOMAN [UNBOX]

2007 QUICKEN Basic Personal Finance Software New In Box

Braun 8995360 Complete Men's Shaver BRAND NEW SEALED! !

Braun 8995360 Complete Men's Shaver BRAND NEW SEALED!

New* TMX ELMO Tickle Me Elmo TM X Seled English Ver

LOVELY by SARAH JESSICA PARKER 3.4 OZ EDP WOMAN [UNBOX]

Ipod Shuffle 1 GB Blue 2nd Second Gen NEW in box+EXTRAS
149.99 BIN has Ink

199.98 Auction has extras

141.99 "Essential Edition" is different type of hard drive

169.99 Auction has extras

ning box version

24.95 Auction has bonus

19.99 Different products

28.95 Auction includes Turbo Tax

165.00 Auction is Mercedes versio

9.99 Auction is unboxed
154.99 BIN includes Ink

39.00 Auction is used

$132.49 \mathrm{BIN}$ is unbranded version

169.99 Auction is unlocked

152.99 Auction has warranty

$132.49 \mathrm{BIN}$ is unbranded versio

195.00 Different colors
65.00 BIN has warranty

65.00 BIN has warranty

195.00 Different colors

132.49 BIN is unbranded version

14.99 BIN in 2005 version

29.99 Auction is purple
195.00 Different colors

$132.49 \mathrm{BIN}$ is unbrand

19.99 Auction is used

19.99 Auction includes UPC (for rebate)

198.99 Auction is unlocked
$132.49 \mathrm{BIN}$ is unbranded version

29.99 Auction is purple orchid

175.00 Auction has warranty

175.00 Auction has warranty

195.00 Different colors

29.88 BIN is tester

25.00 Auction has the Top Secret Box (variation)

25.00 Auction has the Top Secret Box (variation)

380.00 Different products

25.00 Auction has the Top Secret Box (variation)

315.00 Auction is coach e

19.95 Auction includes UPC (for rebate)

65.00 BIN has warranty

315.00 Sidekick 3 and Sidekick ID are different types of phone

29.99 Auction is purple orchic
175.00 Auction has warranty

$132.49 \mathrm{BIN}$ is unbranded version

154.99 "Essential Edition" is different type of hard driv

$132.49 \mathrm{BIN}$ is unbranded version
70.99 Different item numbers

195.00 Different colors

29.99 Auction is purple orchid

29.99 Auction is purple orchid

$132.49 \mathrm{BIN}$ is unbranded versio
70.99 Different item numbers

$27.99 \mathrm{BIN}$ is unboxed

19.99 Auction does not include UPC (for rebate)

145.008985 vs 8995

145.008985 vs 8995

32.99 Auction has the Top Secret Box (variation)

$27.99 \mathrm{BIN}$ is unboxed

29.99 Auction is purple orch 
NEW BRAUN 8985360 COMPLETE ELECTRIC SHAVER RAZOR 8995 NEW BRAUN 8985360 COMPLETE ELECTRIC SHAVER RAZOR 8995 NEW BRAUN 8985360 COMPLET ELECTRIC SHAVER RAZOR T-Mobile Sidekick 3 (T-Mobile) Brand New - No Reserve*
LOVELY SARAH JESSICA PARKER 3.4 oz EDP NIB NO RESERVE

政

TESNITY by Calvin Klein 3.4 oz EDP perfume *SEALED*
SESAME STRET TICKLE ME ELMO WARNING TMX EXTREME DOLL Lovely Sarah Jessica Parker 100ml Women Perfume NIB

NOKIA N93 GSM SIDEKICK III TMOBILE T-MOBILE NR MUSTSE

ETERNITY by Calvin Klein 3.4 oz edp Perfume SEALED NIB

NEW MOTOROLA V3I RAZR BLACK \& GOLD UNLOCKED LIMITED

ETERNITY by Calvin Klein 3.4 oz. EDT PERFUME WOMEN Seal

NEW BRAUN 8985360 COMPLETE ELECTRIC SHAVER RAZOR 8995 NEW BRAUN 8985360 COMPLETE ELECTRIC SHAVER RAZOR 8995

**BRrand New** T-Mobile Sidekick 3 w/car charger!!!
Apple iPod shuffle Second Gen. Pink (1 GB) MP3 NEW
XBOX360 WIRELESS Controller

Western Digital My Book 2 gen silver w/cover

LOVELY SARAH JESSICA PARKER PERFUME 3.4 OZ SEALED

T-Mobile Sidekick 3 NEW Free Shipping No Con $3.40 Z$ III

TEXAS INSTRUMENTS TI-89 TITAN

Apple Ipod Nano Mercedes-Benz Edition (4GB), Silver NEW
GARMIN \$350 STREETPILOT c320 GPS BRAND NEW, V8 MAPS, NR

Apple Ipod Shuffle-1 Gig-Pink

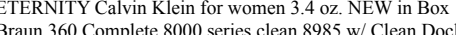

Braun 360 Complete 8000 series clean 8985 w/ Clean Dock
CALVIN KLEIN ETERNITY EDP WOMENS COLOGNE 3.4 OZ. NIB

ETERNITY by Calvin Klein 3.40z EDP women New In Box

Sidekick iD T-Mobile NIB \& extra Dark Gray Bumper!

ORANGE Apple 1GB 2nd Generation iPod Shuffle

USED BELKIN WRSELESS G ROUTER 802.11

AVER RAZOR 8995

TRIC SHAVER RAZOR 8995

NOKIA N93 WORLD CELL PHONE. UNLOCKED. BN NO RESERVE! rand new side kick 3

BASIC - NIB !! NR !'- Includes UPC

X 360 WIRELESS Controller

NEW GARMIN STREETPILOT C320 COLOR GPS CAR NAVIGATION V8 NEW Canon SD600 PowerShot Digital Camera SD 60

T-Mobile Sidekick ID (T-Mobile)

Bumble and Bumble Tonic Lotion, 8 ounc
Callaway HX HOT NEW golf balls 6 Doz

Proactive Renewing Cleanser New 4 oz. Exp. 04/08

XBOX 360 WIRELESS Controller

XBOX 360 WIRELESS Controller

*NEW IN BOX* Original Garmin StreetPilot c330 Auto GPS

Bumble and Bumble Tonic Lotion, 8 ounce spray no box

NEW GARMIN STREETTLLOT C320 COLOR GPS CAR NAVIGATION V8

NEW Western Digital 500GB My Book USB Hard Drive $500 \mathrm{~GB}$
Murad Acne Spot Treatment acne/oily skin -NIB-
政 8995360 Complete Men's Shaver BRAND NEW SEALED!! Bann 8995360 Complete Men's Shaver BRAND NEW SEALED

B

T-Mobile Sidekick 3 Brand New Unopened and Accessories

SARAH JESSICA PARKER Lovely EDP $3.4 \mathrm{oz} / 100 \mathrm{ml}$ TST NIB
* ETERNITY PURPLE ORCHID * 3.4*by * CK * Perfume* NIB

Calvin Klein Eternity Men 3.4 oz*NIB* 100\% Authentic

Tickle Me $\sim$ ELMO

T-Mobile Sidekick 3 Jand New Unopened

Nokia N93 black NEW UNLOCKED unbranded +1GB minis

ETERNITY by Calvin Klein 3.4 COLOGNE SP MEN NEW IN BOX

NEW MOTOROLA RAZR V3i GOLD D\&G DOLCE \& GABBANA MP3 V3

* ETERNITY PURPLE ORCHID * 3.4 * by * CK * Perfume* NIB

Braun 8995360 Complete Powe-Comb *TOP-OF-THE-LINE**

Braum 8995360 Complete Power-Comb *TOP-OF-THE-LINE*

*** Sidekick 3 GSM T-Mobile Bluetooth ** NEW IN BOX

Apple iPod Shuffle 1GB New Apple 1 yr Limited Warranty

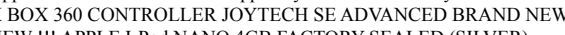

LOVELY by SARAH JESSICA PARKER 3.4 OZ EDP WOMAN [UNBOX] T-Mobile Sidekick 3 Brand New Unopened and Accessoric

X BOX 360 CONTROLLER JOYTECH SE ADVANCED BRAND NEW

SB

New Garmin StreetPilot c330 Portable Car GPS Navigator

Apple iPod Shuffle 1GB New Apple 1 yr Limited Warranty
* ETERNITY PURPLE ORCHID * 3.4*by * CK * Perfume* NIB

Braun electric 360 complete razor NEW

Calvin Klein Eternity Men $3.4 \mathrm{oz} * \mathrm{NIB}^{*} 100 \%$ Authentic

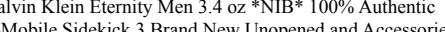

Apple iPod Shuffle 1GB New Apple 1 yr Limited Warranty

Belkin (F5D7230-4) 54Mbps Wireless G Router

ND NEW SEALED!

*** Sidekick 3 GSM T-Mobile Bluetooth ** NEW IN BOX **

NIB intuit Quicken basic 2005 half price of 2006\& 2007
Nokia N93 black NEW UNLOCKED unbranded + 1 GB minisD+more

T-Mobile Sidekick ID (T-Mobile) Brand New

X BOX 360 CONTROLLER JOYTECH SE ADVANCED BRAND NEW

* Sidekick 3 GSM T-Mobile Bluetooth ** NEW IN BOX **

New Garmin StreetPilot c 330 Portable Car GPS Navigator

Canon Powershot SD90 Digital ELPH 10 MEGAPIXEL

** Sidekick 3 GSM T-Mobile Bluetooth ** NEW IN BOX *

8 oz. Bumble and Bumble Tonic Lotion. NEW.

列

C

$X$ BOX 360 CONTROLLER JOYTECH SE ADVANCED BRAND NEW
X BOX 360 CONTROLLER JOYTECH SE ADVANCED BRAND NEW

Garmin StreetPilot c550 GPS Navigator/Traffic Receiver

8 oz. Bumble and Bumble Tonic Lotion. NEW.

NIB Garmin StreetPilot c550 GPS Vehicle Navigator

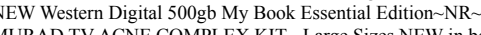

145.008985 vs 899

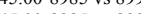

145.008985 vs 8995

339.99 BIN has accessorie

29.99 Auction is purple orch

28.99 Perfume vs cologne
25.00 Auction has Warning box version

339.99 BIN has accessori

$591.00 \mathrm{BIN}$ includes $1 \mathrm{~GB}$ miniSD+more

31.99 Perfume vs cologne

29.99 Auction is purple orchid

29.99 Auction is purple orchid

142.958985 vs 8995

132.95 30.00 Auction vas car charge

65.00 BIN has warranty

19.85 Different brands

154.99 "Essential Edition" is different type of hard drive

$27.99 \mathrm{BIN}$ is unboxed

339.99 BIN has accessorie

98.99 Auction is opened
19.85 Different brands

172.99 Auction is Mercedes version

264.99 Different product

65.00 BIN has warranty

29.99 Auction is purple orchid

100.00 Auction has "Clean D

28.99 Women vs men

339.99 Sidekick 3 and Sidekick ID are different types of phones

65.00 BIN has warranty

29.95 Auction is used

145.008985 vs 8995

330.00 Auction has extra battery

$14.99 \mathrm{BIN}$ in 2005 version

591.00 BIN has "minisD+mor"

different types of phones

idekick ID are different types of phone

(300.00 Dierent products

.00 Sidekick 3 and Sidekick ID are different types of phones

has "no box"

200.00 "Hot" and "Tour" are different type of golf balls

15.99 Different expiration dates

19.85 Different brand

19.85 Different brand

17.49 Auction has "no box"

19.99 "Essential Edition" is different type of hard drive 